\title{
Reliability of Free Radial Forearm Flap for Tongue Reconstruction Following Oncosurgical Resection
}

\section{Gaurab Ranjan Chaudhuri ${ }^{1}$}

\section{Introduction}

\section{$\underline{\text { ABSTRACT }}$}

Primary closure following oncosurgical resection of carcinoma tongue has been found to compromise tongue function in regards to speech and swallowing very badly. In contrast, reconstruction of tongue with free radial forearm flap following oncosurgical resection has shown promising functional outcome.

\section{Materials and Methods}

Thirteen patients (ten male and three female) with squamous cell carcinoma involving anterior 2/3rd of tongue had undergone either hemiglossectomy or subtotal glossectomy. Reconstruction was done with free radial forearm flap following oncosurgical resection and neck dissection. All of them received postoperative radiotherapy. Follow-up ranged from 2 months to 2 years. The age of the patients ranged between 32 and 65 years. Flap dimension ranged from $7 x 6 \mathrm{~cm}$ to $10 x 8 \mathrm{~cm}$. Vascular anastomosis performed in an end-to-end manner with 8-0 Ethilon ${ }^{\circledR}$ under loupe magnifiacation.

\section{$\underline{\text { Results }}$}

Venous congestion occurred in one patient after 48 hours postoperatively and the flap underwent complete necrosis on postoperative day 5. Postoperative hematoma was found in one patient within first 24 hours of reconstruction. Re-exploration was done immediately, blood clots were removed. No fresh bleeding point was seen and the flap survived. In this series, 12 out of 13 flaps survived completely (92\%).

\section{Conclusion}

The free radial forearm flap has become a workhorse flap in head and reconstruction due to its lack of extra bulk, relative ease of dissection, long vascular pedicle, good calibre vessels, malleability and minimal donor site morbidity. Furthermore its low flap loss and complication rate offer the best choice for tongue reconstruction.

$\underline{\text { Kevwords }}$

Carcinoma, Squamous Cell; Tongue; Glossectomy; Reconstructive Surgical Procedures; Free Tissue Flaps; Forearm; Radial Artery; Ulnar Artery

$\mathrm{S}$ quamous cell carcinoma of tongue is a highly malignant condition and results in significant mortality and morbidity. However, early detection and treatment results in better outcome and prolongs survival. The morbidity following extensive resection of tongue results in severe speech and swallowing disturbances and life threatening aspiration that needs reconstruction.

The ideal method of reconstruction should provide good functional and aesthetic outcome. The field of reconstructive surgery in the head-neck region is a dynamic one and has gone through numerous changes in past two decades. Microvascular free flap technique has largely replaced other techniques. Free flaps such as radial forearm flap and anterolateral thigh flap have become the first choice in tongue reconstruction. Preservation of function including speech and swallowing and restoration of appearance are the major goals of reconstruction.

Our technique of free radial forearm flap for reconstruction of tongue following oncosurgical resection in a series of patients could fulfil the reconstructive goals to a great extent.

1 - Department of Plastic Surgery, R G Kar Medical
College, Kolkata
Corresponding author:
Dr Gaurab Ranjan Chaudhuri
email: gaurabchaudhuri@rediffmail.com


The free radial forearm flap is a workhorse flap in tongue reconstruction. Its lack of extra bulk, robust vascularity, long vascular pedicle, good calibre of the vessels, relative ease of dissection, flexibility in designing and lack of significant donor site morbidity are among its main advantages. Its low flap loss and complication rate offer the best choice for reconstruction of tongue following oncosurgical resection.

\section{Materials and Methods}

This paper included thirteen patients presented with proliferative or ulceroproliferative growth (squamous cell carcinoma as confirmed preoperatively by biopsy and histopathological examination) involving anterior $2 / 3^{\text {rd }}$ of tongue who were operated between January, 2013 to March, 2015 (Figs. 1, 2); reconstructed with free radial forearm flap (harvested from left side in all cases) following oncosurgical resection (either hemiglossectomy or subtotal glossectomy) and neck dissection; all of them received postoperative radiotherapy; ten male patients and three female patients were included in this study. The age of our patients ranged between 32 and 65 years. Flap dimension ranged from $7 \times 6 \mathrm{~cm}$ to $10 \times 8 \mathrm{~cm}$.

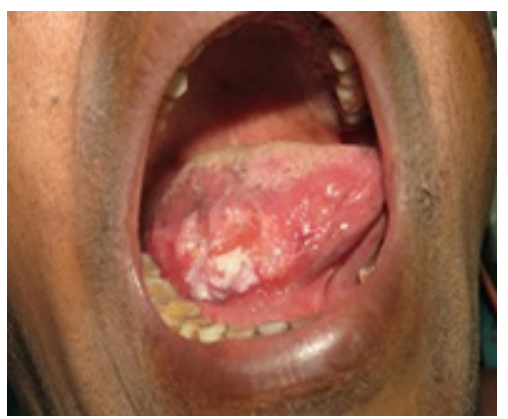

Fig. 1 Ulceroproliferative mass involving right lateral border of tongue

All patients were subjected to:

- Thorough history taking and clinical examination (including Allen's Test)

- Preoperative laboratory investigations

- Evaluation of coagulation profile

- CT scan of tongue, floor of the mouth and neck

- Doppler flowmetry of the forearm (left in all cases as flap donor site in this study) to assess the status of radial and ulnar arteries.

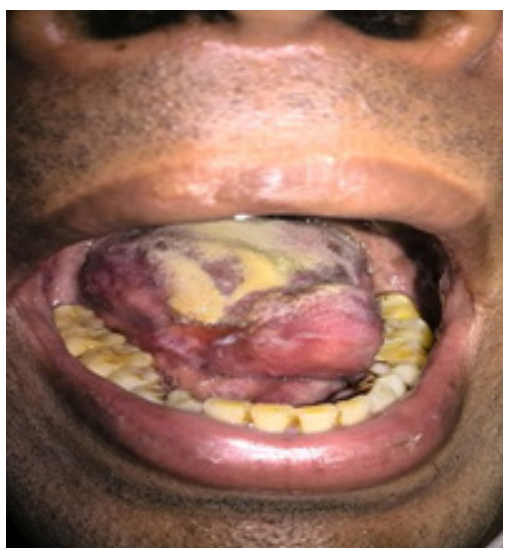

Fig. 2 Ulceroproliferative mass involving whole anterior 2/3rd of tongue

\section{Surgical Technique}

Two teams approach was utilized in all cases to shorten the whole surgical procedure where one team was involved in neck dissection and local resection; and other team harvested the flap at the same time (Figs.3,4).

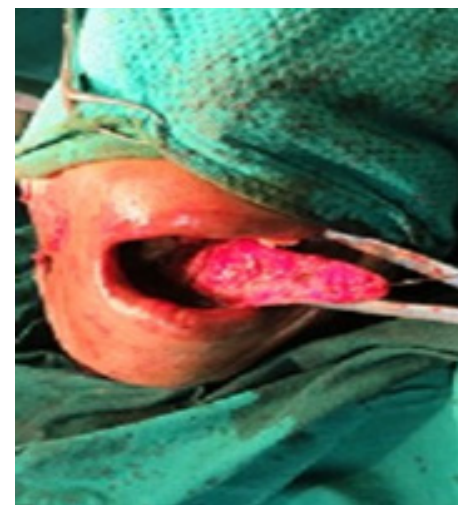

Fig. 3 Post excisional defect

The free radial forearm flap was raised from the distal third of forearm in subfascial plane from distal to proximal direction keeping the paratenon of the underlying tendon intact and preserving the septum in between brachioradialis and flexor carpi radialis muscles meticulously; the superficial venous system 


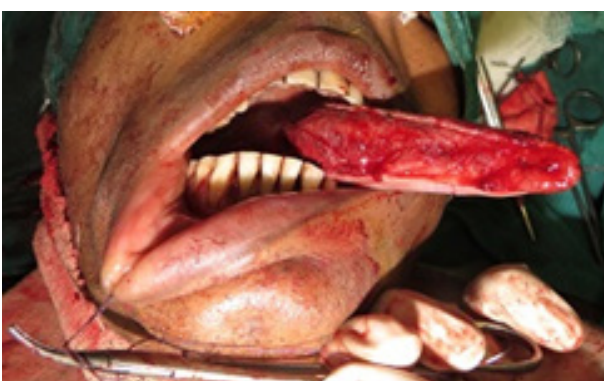

Fig. 4 Post excisional defect

was preserved; the cephalic vein and vascular pedicle of the flap dissected up to the cubital fossa to gain a long pedicle that aided in tension-free anastomosis in the neck (Figs. 5,6,7).

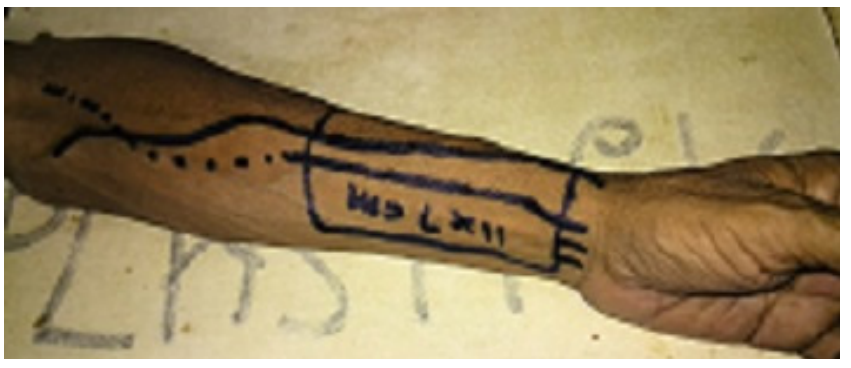

Fig. 5 Preoperative flap marking done over the donor site

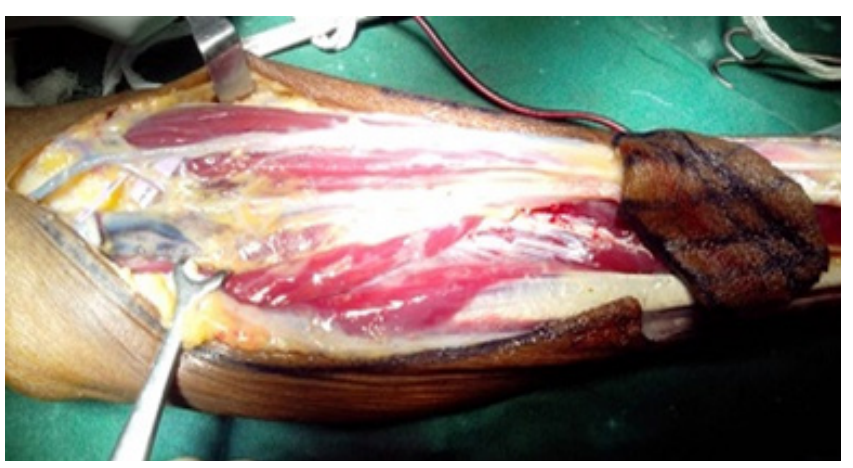

Fig. 6 Flap elevated off the donor site connected only by the vascular pedicles

One suitable recipient artery (facial artery or superior thyroid artery in our cases) and two veins (external jugular vein and common facial vein) were chosen in the neck; After partial flap inset with few anchoring sutures with the remaining part of the tongue and floor

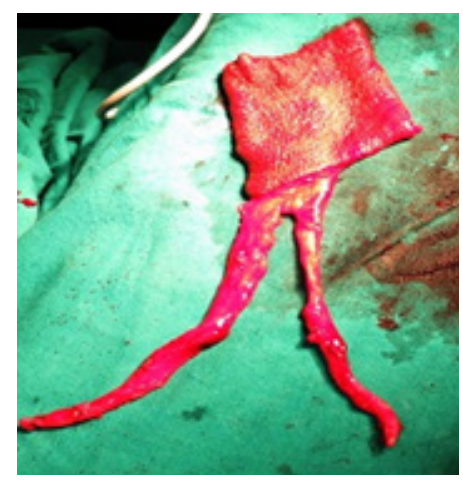

Fig. 7 Flap completely detached from the donor site with division of its vacular pedicles

of the mouth, vascular anastomosis performed in an end-to-end manner with 8-0 Ethilon ${ }^{\circledR}$ (Fig. 8) under loupe magnification in following order:

- Cephalic vein with external jugular vein

- Radial artery with facial artery or superior thyroid artery

- One venae comitantes of radial artey with common facial vein

In our study, we performed double venous anastomosis in 10 patients and single venous anastomosis (one of the venae comitantes of radial artery with common facial vein) in 3 patients.

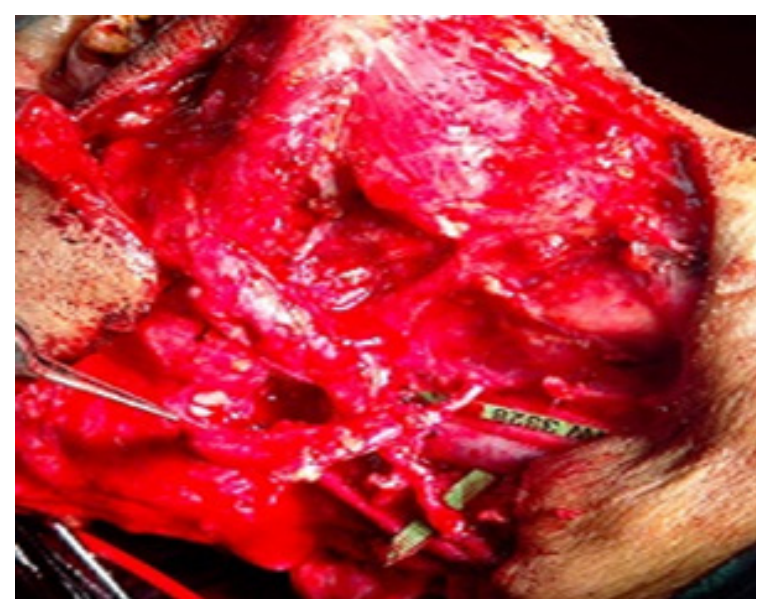

Fig. 8 Vascular anastomoses performed in the neck

Successful anastomosis was confirmed by "milking test' and bleeding observed at the edges of the flap; and then the flap inset was completed. Flap donor site was 
covered with split thickness skin graft harvested from thigh.

\section{Discussion}

Squamous cell carcinoma of tongue typically affects men in sixth to eighth decades having a strong association with alcohol and tobacco abuse over years. Less than $4 \%$ of these lesions have been reported in patients younger than 40 years. Retrospective analysis suggested that the disease runs a more aggressive course in under- 40 age group. Metastatic spread of SCC of the tongue is facilitated by its rich lymphatic net work and tends to increase with the size of the primary tumour. ${ }^{1}$ Approximately, $50 \%$ of tongue cancers present with neck node involvement. In order to achieve a good therapeutic control of the disease, a wide local excision and neck dissection are to be done as a primary procedure. This oncosurgical resection results in a large three dimensional defect of the tongue and floor of the oral cavity that impairs speech, swallowing and imposes risk of aspiration. It is therefore prudent to reconstruct the defect with a well vascularised tissue for a good functional substitution.

The free radial forearm flap is a workhorse flap in tongue reconstruction. Its lack of extra bulk, long vascular pedicle, good calibre of the vessels, pliability and minimal donor site morbidity are among its main advantages; the flap harvest is relatively easy and good calibre of the vessels is suitable for anastomosis with a high success rate. The free radial forearm flap, therefore, offers the best choice for reconstruction of tongue following oncosurgical resection.

In our study, venous congestion occurred in one patient after 48 hours postoperatively and the flap underwent complete necrosis on postoperative day 5; postoperative hematoma was found in one patient within first 24 hours of reconstruction; we immediately went for re-exploration, removed the blood clots and checked for anastomotic leakage or any active source bleeding; but couldn't detect any fresh bleeding point and the flap survived. Thus, our flap survival rate was approximately $92 \%$.

Song et al. ${ }^{2}$ reported a flap survival rate greater than $90 \%$; Kruse et al. ${ }^{3}$ reported that the success rate of free radial forearm flap was more than $95 \%$; Shibahara et al. ${ }^{4}$ reported a total success rate of $100 \%$. It emphasizes the reliability of free radial forearm flap for reconstruction of head and neck defect.

Free radial forearm flap, also known as 'Chinese' flap, is a versatile fasciocutaneous flap was first described by Yang and colleagues in 1981, who used it to treat postburn neck contracture. ${ }^{5}$

Soutar proposed the use of free radial forearm flap for reconstruction of oral cavity in 1983 and thereafter the flap became the most utilized technique for intraoral reconstruction. ${ }^{6}$

Cheng first used this flap for tongue reconstruction. ${ }^{7}$

Khashaba and McGregor demonstrated equal and adequate flow through superficial and deep venous systems of forearm by occluding one or the other system in-situ during flap harvest. ${ }^{8}$

To reduce the donor site morbidity and to improve its aesthetic appearance, Webster and Robinson described free radial forearm flap harvest in suprafascial plane. ${ }^{9}$

Many surgeons compared the postoperative swallowing functions of patients who underwent reconstruction of tongue with free radial forearm flap with patients who underwent primary closure of post excisional defect and concluded that the flap added bulk, thus improved pharyngeal clearance and deglutition. Patients with primary closure were unable to lift the tongue tip, had poor tongue to palate contact during initiation of swallowing and premature spilling of bolus into the pharynx.

Some surgeons retrospectively compared the postoperative speech and swallowing in patients who underwent hemiglossectomy for carcinoma of anterior tongue. Immediate reconstruction was done with free radial forearm flap in $50 \%$ patients and other $50 \%$ were reconstructed with free anterolateral thigh flap. The functional outcome with both flaps were adequate and the two groups did not differ significantly for either speech or swallowing.

Some surgeons advocate an innervated FRFF in an attempt to produce a more favourable functional outcome; however, a number of studies have shown that very little and insignificant benefit is achieved when compared to an insensate flap. ${ }^{10,11}$ 
In this study, we used free radial forearm flap in a series of 13 patients with squamous cell carcinoma of tongue following oncosurgical resection with satisfactory aesthetic and functional outcome in regards to speech and swallowing (Figs. 9,10,11,12,13) with minimal donor site morbidity.

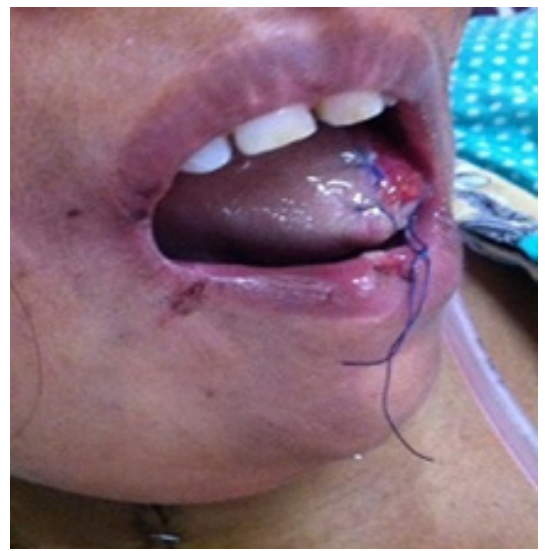

Fig. 9 Postoperative 2nd week

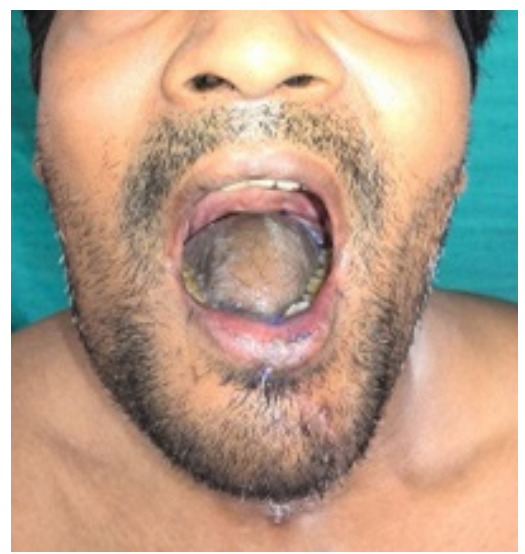

Fig. 10 Postoperative 2nd month

\section{Conclusion}

Compared with primary closure of the post-excisional defect of the tongue, it is better to reconstruct the defect with free radial forearm flap when $50 \%$ or more tongue is resected. The overall satisfaction of the patients in regards to function and cosmesis in tongue reconstruction was high when using free tissue transfer in the form of free radial forearm flap following wide local oncosurgical resection. Although microvascular reconstruction is a lengthy procedure and requires

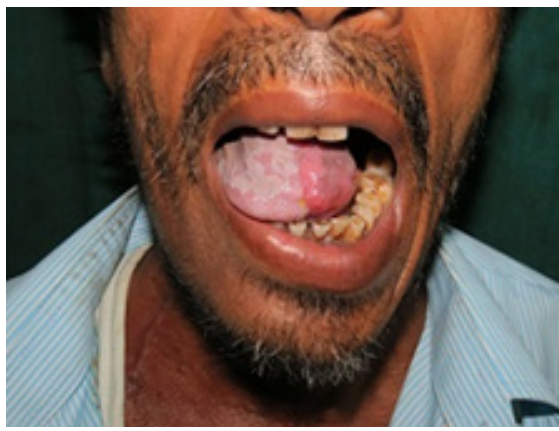

Fig. 11 Postoperative 6th month

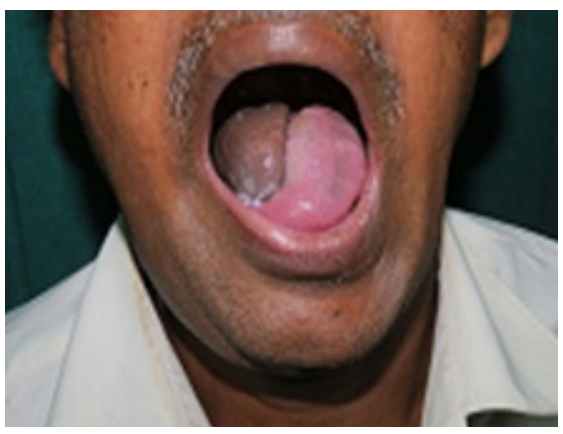

Fig. 12 Postoperative 2nd year

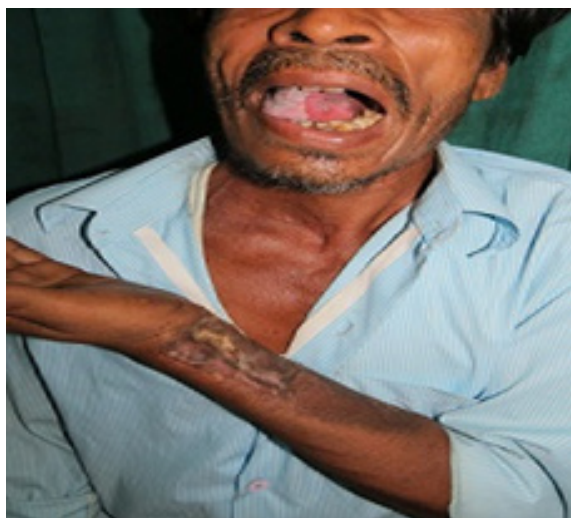

Fig. 13 Complete skin graft taken over the donor site utmost surgical skill and expertise, the post-excisional morbidity is reduced to a great extent while maintaining the function of the important organ like tongue.

\section{References}

1. Popovtzer A. Squamous cell carcinoma of the oral tongue in young patients. Laryngoscope 2004; 114:915-7

2. Song R, Gao Y, Song Y, et al. The forearm flap. Clin.Plast. 
Surg. 1982; 9:21-6

3. Kruse AL, Bredel MG, Lubbers HT, Jacobsen C, Gratz KW, Obwegeser JA. Clinical reliability of radial forearm free flap procedure in reconstructive head and neck surgery. J Craniofac Surg $2011 ; 22: 822-5$

4. Shibahara T, Mohammed AF, Katakura A, Nomura T. Long-term results of free radial forearm flap used for oral reconstruction: functional and histological evaluation. J Oral MaxillofacSurg 2006; 64:1255-60

5. Yang GF, Chen PJ, Gao YZ, et al. Forearm free skin flap transplantation: A report of 56 cases. Br.J.Plast.Surg. 1997;50:162-5

6. Soutar DS, Scheker LR, Tanner NSB, McGregor IA. The radial forearm flap: A versatile method for intraoral reconstruction. Br.J.Plast.Surg. 1983; 36:1
7. Cheng BS. Free forearm flap transplantation in repair and reconstruction of tongue defects. Chung Hua Kon Chiang Tsa Chih. 1983;18-39

8. Khashaba AA, McGregor IA. Haemodynamics of the radial forearm flap. Br.J.Plast.Surg. 1986; 39:441-50

9. Webster HR \& Robinson DW. The radial forearm flap without fascia and other refinements. Eur J Plast Surg, 1995;18:11-3

10. Kuriakose MA, Loree TR, Spies A, et al. Sensate radial forearm free flaps in tongue reconstruction. Arch Otolaryngol Head Neck Surg 2001;127: 1463-6

11. Netscher D, Armenta AH, Meade RA, et al. Sensory recovery of innervated and non-innervated radial forearm free flaps: functional implications. J Reconstr Microsurg 2000; 16: $179-85$ 